\title{
Applicability of the classical molecular dynamics method to study X-ray irradiated molecular systems
}

\author{
Z Jurek $^{1,2}$, B Ziaja ${ }^{1,2,3}$ and R Santra ${ }^{1,2,4}$ \\ ${ }^{1}$ Center for Free-Electron Laser Science, Deutsches Elektronen-Synchrotron, \\ Notkestrasse 85, D-22607 Hamburg, Germany \\ ${ }^{2}$ Hamburg Centre for Ultrafast Imaging, Luruper Chaussee 149, 22761 Hamburg, \\ Germany \\ ${ }^{3}$ Institute of Nuclear Physics, Polish Academy of Sciences, Radzikowskiego 152, \\ 31-342 Kraków, Poland \\ ${ }^{4}$ Department of Physics, University of Hamburg, Jungiusstrasse 9, 20355 Hamburg, \\ Germany \\ E-mail: zoltan.jurek@desy.de
}

\begin{abstract}
.
Classical molecular dynamics (MD) has been successfully applied to model radiation-induced dynamics of highly ionized assemblies of atoms, including the dynamics of electrons, released during ionization processes. Here we test the applicability of a classical molecular dynamics scheme in a yet unexplored regime: for a strongly bound molecular system represented by buckminsterfullerene, $\mathrm{C}_{60}$, singly ionized by an X-ray pulse. We show MD simulation results obtained for electron and ion spectra, and compare them to existing experimental data from synchrotron experiments. We identify the sources of discrepancies between the classical simulations and experiments, and discuss possible improvements of the model. Our calculations establish limits for the applicability of classical MD simulations to X-ray irradiated systems. These classical simulations are much more computationally efficient than any rigorous quantum calculations, and are, therefore, often the only option. The conclusions obtained can be useful for planning computational studies of irradiated large molecular assemblies.
\end{abstract}

PACS numbers: 31.15.xv; 61.48.-c; 33.60.+q 


\section{Introduction}

The classical molecular dynamics (MD) method was originally developed to study the dynamics of atoms in neutral assemblies by applying force fields to describe chemical bonds and integrating Newton's equations of motion for the constituent atoms. In later studies, in addition to modeling the dynamics of atoms and ions, the method started also to be used for modeling the dynamics of fast electrons. In particular, it was used to simulate the ionization of finite-size samples, irradiated by intense optical laser pulses $[1,2,3,4,5]$.

MD methods usually treat ionization stochastically by assigning corresponding rates to the specific ionization processes. When an ionization occurs, a classical free electron is released. This electron is then propagated together with the atoms, ions and other free electrons. As an optical field (wavelength $\sim 800 \mathrm{~nm}$ ) ionizes the atoms mainly via field ionization and then heats the electrons efficiently via inverse bremsstrahlung, this classical treatment of the particle dynamics is adequate.

In 2005, FLASH [6], the first soft X-ray free-electron-laser (FEL), and in 2009, LCLS [7], the first hard X-ray FEL, started user operation. Their intense X-ray radiation enables creating highly ionized states of matter. During the last decade various models have been developed to reveal the dynamics driven by high intensity X-ray pulses $[8,9,10,11,12,13]$. They are based on the classical description of the real space dynamics, although the predominant ionization mechanisms, outer- and innershell ionization with the subsequent Auger decay, were entirely different from those observed in the optical regime.

However, the question arises as to whether the classical approach is applicable under conditions of X-ray irradiation. The classical MD scheme has proved to be successful in the case of the irradiation of atomic or molecular assemblies with intense X-ray pulses, when the interatomic bonds break early in the exposure, and the irradiated system then quickly turns into plasma [14]. However, it is not at all clear if this approach can be used when only a few atoms within a strongly bound molecular system are ionized by a low-intensity X-ray pulse. In what follows we investigate this question with our MDbased tool, XMDYN [15]. We apply it to model the evolution of buckminsterfullerene $\left(\mathrm{C}_{60}\right)$ molecules irradiated by synchrotron light.

Buckminsterfullerene $\left(\mathrm{C}_{60}\right)$ consists of 60 carbon atoms. Each carbon atom in the structure is bonded covalently with 3 others, forming pentagonal and hexagonal carbon rings. The diameter of a $\mathrm{C}_{60}$ molecule is $\sim 7.1 \AA$, the nearest neighbor distance of the carbon atoms is $\sim 1.45 \AA$. The total potential energy due to the bonds is $\sim 430 \mathrm{eV}$ $(\sim 4.8 \mathrm{eV} /$ bond $)$. The ionization potential is $\sim 7.6 \mathrm{eV}[16]$. The $\mathrm{C}_{60}$ molecule is extraordinarily stable, and can withstand both high pressures and high temperatures [17]. The exposed surface of the structure can selectively react with other species while maintaining the spherical geometry [17]. Also, atoms and small molecules can be trapped inside the $\mathrm{C}_{60}$ cage without reacting [17].

The small size and, at the same time, the extreme stability of $\mathrm{C}_{60}$ due to the strong 
bonding between carbon atoms make it an ideal object to study the applicability of the classical MD technique. The relatively small number of involved particles enables a fast computation. In what follows we investigate the applicability of classical molecular dynamics scheme for $\mathrm{C}_{60}$, singly photoionized with soft $\mathrm{X}$-ray radiation of energies between $290 \mathrm{eV}$, i.e., above the K-edge for carbon, and $800 \mathrm{eV}$. Considering typical synchrotron pulse parameters (fluence of $\sim 10^{4} \mathrm{ph} / \mu \mathrm{m}^{2} /$ pulse [18]) and the photoionization cross section of carbon $(<1 \mathrm{Mb})$, the average number of photoionizations within $\mathrm{C}_{60}$ during a pulse is $\lesssim 10^{-4}$. Therefore single photoionization of the molecule is much more probable than multiple photoionization, and it gives the dominant contribution to the measured signal.We show simulation results for electron and ion spectra obtained after the irradiation of $\mathrm{C}_{60}$, and compare them to existing data from synchrotron experiments. Beside the reasonable agreement, we find some discrepancies between the simulations and experiments, mainly due to the specific model chosen for describing electron impact ionization (collisional ionization). We discuss its possible improvement. The conclusions obtained can be useful for designing computationally efficient tools for simulation of X-ray irradiated molecular assemblies.

\section{Dynamics of $\mathrm{C}_{60}$ after single photoionization}

We follow the time evolution of the $\mathrm{C}_{60}$ molecule, initiated by a single photoabsorption event. If the energy of the incoming photon lies below the $1 s$ threshold $(\sim 290 \mathrm{eV})$, only electrons from the L-shell are excited. If the photon energy is higher that the $1 \mathrm{~s}$ threshold, inner-shell (K-shell) photoionization is predominant. The remaining K-shell vacancy then relaxes through Auger electron emission. In a molecular environment the resulting two valence holes are finally located at different atoms [19, 20].

The photo- and Auger electron emission is often accompanied by ejection of further low energy electrons (e.g., shake-off processes [21] or double Auger decay [21]). However, within the molecular environment this process cannot be uniquely distinguished from a single photoionization or an Auger decay followed by an impact ionization process. Therefore, in our approach we model these processes by subsequent impact ionizations initiated by primary photoelectrons and Auger electrons. The secondary electrons released further ionize the molecule. $\mathrm{As}_{\mathrm{s}} \mathrm{C}_{60}$ is small, most of the electrons can leave it after one interaction or even without interacting with carbon atoms or ions in the molecule. The increased charge within the molecule then leads to interatomic Coulomb repulsion. If the charge is high enough, it can lead to bond breaking and molecule fragmentation.

Our modeling tool, XMDYN [15] (extension of the original framework presented in Ref. [9]) describes the sample as an assembly of isolated atoms, but it can treat some molecular effects. Atomic ions and free electrons are treated as classical particles. Their dynamics are followed in full real space under open boundary conditions. Coulomb forces between charged particles are taken into account. Chemical bonds (intramolecular 
forces) between neutral carbon atoms are described with the Brenner force field (with parameter set for 'potential I') [22, 23], an empirical potential developed for hydrocarbons, diamond and fullerenes. We assume that these bonds break between two charged ions. As a bond breaks, we set the pair potential contribution to the manybody Brenner force field from the two charged ions to be zero $\left(f_{i, j}(r)=0\right.$, if $0<q_{i}$ and $0<q_{j}$, using the notation of Ref. [22]). The non-relativistic classical equations of motion are integrated with a 0.8 as-short timestep (relative error of energy conservation is less than $0.1 \%$ ). The configurations of all atoms, i.e., the occupation numbers of different $(n, l)$ orbitals, and their changes due to the ionization are tracked. The Monte Carlo method is used to model photoionization and Auger decays. Probabilities of these processes are calculated with the corresponding quantum-mechanical cross sections and rates from the XATOM package [24]. A specific scattering channel is then chosen accordingly, in a random way, during each realization of the $\mathrm{C}_{60}$ photoionization. Note that these cross sections describe the interaction of isolated carbon atoms or ions with the incoming photons. Molecular Auger decay is realized through filling the K-shell vacancy by an L-shell ( $2 \mathrm{~s}$ or $2 \mathrm{p}$ ) electron from the photoionized atom, and transferring the excess energy to an L-shell electron from one of the neighboring atoms.

The electron impact ionization rate depends on the atomic configuration and the kinetic parameters of the impact electron at its closest approach to the carbon atom or ion [9]. To model impact ionization, we use either the atomic cross sections calculated for isolated atoms and ions [25], or the cross sections for electron impact ionization within crystalline diamond, obtained from band-structure calculations [26], including plasmon contributions [27] (Figure 1). The results obtained with both models are then compared and discussed.

In order to obtain statistically reliable predictions for the electron spectra and ion yields, many trajectories (initialized with a different random seed) of singly photoionized $\mathrm{C}_{60}$ (including the photoelectron) are followed. The simulation time (up to $200 \mathrm{ps}$ after the photoionization) was long enough to capture all ionization events: Auger decay - within tens of femtoseconds after the photoionization - and any following impact ionizations. In more than $98 \%$ of the trajectories no fragmentation occurred during the propagation. During each simulation the evolution of the photoionized system is recorded including, e.g. information on the emitted electron spectra and charge states. After averaging over the number of realizations (typically 1500 trajectories), the results are compared to experimental data.

Figure 2 shows the calculated electron spectra compared to experimental results by A. Reinkoester et al. [21] from single photoionization of $\mathrm{C}_{60}$ by a $390 \mathrm{eV}$ photon. We normalized the theoretical and experimental spectra (in arbitrary units) in such a way that the areas under the curves between $200 \mathrm{eV}$ and $300 \mathrm{eV}$, which are proportional to the number of emitted Auger electrons, equal each other.

Within these theoretical spectra one can identify the following contributions: (i) the outer-shell photoelectron peak, located at an energy close to the incoming photon energy 




Figure 1.

(Color online) Atomic [25] and diamond [26] impact ionization cross sections used in the calculations.

(tiny peak; not shown), (ii) individual Auger lines, corresponding to the Auger electron emission from various outer shells of carbon $(\sim 250-300 \mathrm{eV})$, (iii) 1 s photoelectron peak from the K-shell photoemission (the strongest line), and (iv) the distribution of low energy secondary electrons. The broadening of the photoelectron line is due to the energy loss during impact ionization events. The Auger lines are broadened for the same reason. In addition, they are slightly shifted towards higher energies when compared to the isolated carbon case, due to the energy lowering of the available Auger channels as a consequence of the separation of the two final-state holes on different atomic sites.

The experimental and theoretical spectra are in a fairly good agreement with each other. Three discrepancies can be identified: (i) Discrete Auger lines appear in the theoretical spectra while they are missing in the experimental specta. This is a consequence of the atomistic approach applied, i.e., a finite number of discrete Auger energies has been used in the simulation while in a real $\mathrm{C}_{60}$ molecule the Auger energies have a continuous distribution. However, the overall contribution of the narrow lines to the spectrum is small. (ii) There is a $\sim 10 \mathrm{eV}$ gap near the theoretical K-shell photoelectron peak. This is due to the fact that electronic excitations without ionization 



Figure 2.

(Color online) Electron emission spectra as a function of the electron energy for $\mathrm{C}_{60}$ molecules (in gas phase) after single photoionization with a photon of energy: (a),(b) $390 \mathrm{eV}$ and (c),(d) $800 \mathrm{eV}$. Theoretical spectra were obtained from classical MD simulations with XMDYN [15] and are compared to the available experimental data [28]. The theoretical spectra in panels (a),(c) were obtained with electron impact ionization cross sections for isolated ions and atoms, the theoretical spectra in panels (b),(d) were obtained with electron impact ionization cross sections within crystalline diamond.

(with the energy transfer less than the minimal electron binding energy) are not included in the model. (iii) The spectra differ at low electron energies. This is the regime in which the spectra are dominated by the secondary electrons, created during impact ionization processes. They are strongly influenced by the specific parametrization of the electron impact ionization cross sections. As expected, at low electron energies the atomic cross sections for collisional ionization are not accurate, as molecular (band-structure) effects strongly influence the electron impact ionization processes. Consistently, the simulations using diamond electron impact ionization cross sections (Figure 2.b) are in better agreement with the data, as the environmental effect is then included in the cross section. 
We also show theoretical predictions for electron spectra at a photon energy of $800 \mathrm{eV}$. To our knowledge, no measurements have been published at this energy. The theoretical spectra (Figure 2.c-d) obtained with different electron impact ionization cross sections show a good mutual agreement for high energy electrons. They differ again at low energies.

We conclude that the correct parameterization of the electron impact ionization cross section is crucial for an accurate description of the molecular dynamics. This finding is confirmed by a comparison of $\mathrm{C}_{60}$ ion yields, obtained from our simulations, to the experimental data from [28]. Figure 3 shows ion yields obtained at incoming photon energies between 280 and $800 \mathrm{eV}$. They are compared to the available experimental data, recorded between the $1 s$ threshold and $340 \mathrm{eV}$. Note that we compare our predictions to the total yield of $\mathrm{C}_{60}^{+q}$ and its fragments, e. g. $\mathrm{C}_{58}^{+q}$, as our model does not describe the ejection of neutral carbon dimers.

Our simulations allow us to identify the ionization channels leading to the production of various $\mathrm{C}_{60}$ ions. Singly charged ions, $\mathrm{C}_{60}^{+1}$, result from a rare L-shell (outer-shell) photoionization event of one of the constituent carbon atom. Doubly charged $\mathrm{C}_{60}^{+2}$ are created after the predominant $\mathrm{K}$-shell (inner-shell) ionization of a carbon atom, followed by a subsequent Auger emission. The more highly charged ions, $\mathrm{C}_{60}^{+3}$, can be created mainly after K-shell photoionization, with a K-shell electron and an Auger electron emitted, and an impact ionization by one of these electrons.

The obtained theoretical ion yields follow the trends observed in the experimental ion yields. The predictions, using electron impact ionization cross sections for isolated atoms distinctly overestimate the number of the ionization events at a low-energy photon impact. The reason for this is that in this photon energy regime, K-shell photoelectrons that cause impact ionization have low $(<100 \mathrm{eV})$ energies. At such energies the impact ionization cross sections for isolated atoms are inaccurate in molecular environment. To compare, we performed an alternative calculation, using the cross section for the impact ionization within diamond [26]. The results obtained are in better agreement with the data, in particular, when comparing ion yields for doubly- and triply ionized $\mathrm{C}_{60}$ after the irradiation of $\mathrm{C}_{60}$ with photons of energies, $290-320 \mathrm{eV}$.

Let us mention that we performed also additional simulations using the atomic impact ionization cross sections corrected for molecular effects with the method proposed in [29]. However, in our case this did not lead to any significant improvement of theoretical electron and ion spectra, when comparing the 'uncorrected' and 'corrected' results to the experimental data (not shown).

\section{Conclusions}

In summary, we performed classical MD calculations for electron spectra and ion yields obtained from single $\mathrm{X}$-ray-photon absorption by a $\mathrm{C}_{60}$ molecule and compared 

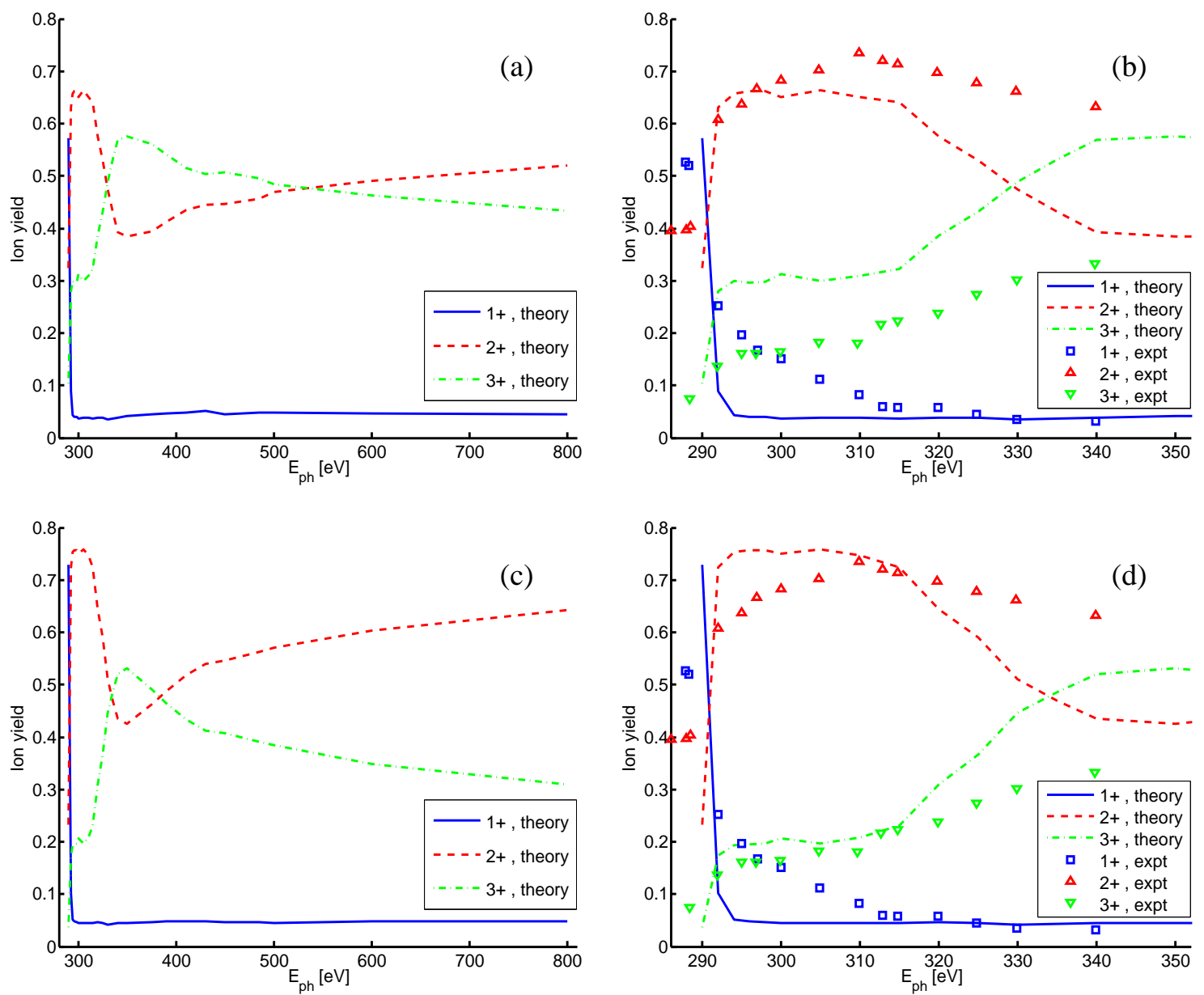

Figure 3.

(Color online) Normalized $\mathrm{C}_{60}$ ion yields $\left(\mathrm{C}_{60}^{+q}\right.$ for $\left.q=1,2,3\right)$ obtained from our classical MD model of photoionized $\mathrm{C}_{60}$ as a function of the energy of the incoming photon: (a),(c) spectra within 280-800 eV energy range; (b),(d) zoomed plots, for the energy range between $280-350 \mathrm{eV}$, compared to the experimental data from [28]. The theoretical spectra in panels (a),(b) were obtained with the electron impact ionization cross sections for isolated ions and atoms, and the theoretical spectra in panels (c),(d) were obtained with the electron impact ionization cross sections within crystalline diamond.

them to the available data from synchrotron experiments. The experimental and theoretical electron emission spectra at a photon energy of $390 \mathrm{eV}$ (which yields a K-shell photoelectron energy of $\sim 100 \mathrm{eV}$ ) are in fairly good agreement with each other. That part of the spectra corresponding to the Auger emissions, quite accurately describes the molecular Auger decay. Generally, minor discrepancies at higher electron kinetic energies originate from the final set of available discrete Auger transitions used in the simulation and from the neglect of the low-energy electron excitation processes. The much larger discrepancy at low electron energies is due to the approximate treatment of the impact ionization. As at low electron impact energies this process is strongly influenced by molecular (band-structure) effects, this discrepancy can be partially corrected by 
applying electron impact ionization cross sections for diamond. The low-energy part of the theoretical spectra is then found to be in better agreement with the data, as the environmental effect is included in the cross section.

The effect of a specific parametrization of the impact ionization cross section is even more pronounced at lower photon energies, according to the comparison of the experimental and theoretical $\mathrm{C}_{60}$ ion yields at photon energies between 290 and $340 \mathrm{eV}$ (with photoelectron energy $\lesssim 60 \mathrm{eV}$ ). As more highly charged $\mathrm{C}_{60}$ ions are produced as the result of impact ionization processes, accurate modeling of impact ionization is crucial for obtaining correct predictions for ion yields. Therefore, an approximate way of treating the impact ionization cross sections has a large effect on the predicted ion yields. Nevertheless, they follow the trends observed in the experimental data, and the agreement again improves when the diamond electron impact ionization cross sections are used.

To conclude, for the considered strongly-bound singly-photoionized molecular system, the $\mathrm{C}_{60}$ molecule, we have obtained a surprisingly good agreement of our classical simulations with the experimental data. The main source of discrepancy between theory and data has been identified, as originating from the approximate treatment of the electron impact ionization cross sections. This treatment can be improved in the future by applying an ab-initio method. However, the results obtained allow us to expect that as the energy of the incoming photon increases, so that the impact ionization is caused by photoelectrons of higher energies, it can still be accurately described by atomistic impact ionization cross sections. Our model, even in its present form, can then be expected to accurately address the experimental data. Measurements at higher photon energies will be performed in the near future [30]. They will validate this expectation.

\section{Acknowledgments}

We thank P. Juranic and J. Viefhaus for discussions. This work was supported in part by the excellence cluster 'The Hamburg Centre for Ultrafast Imaging - Structure, Dynamics and Control of Matter at the Atomic Scale' of the Deutsche Forschungsgemeinschaft.

\section{References}

[1] T. Ditmire. Phys. Rev. A, 57:R4094-R4097, 1998.

[2] I. Last and J. Jortner. Phys. Rev. A, 62:013201, 2000.

[3] K. Ishikawa and T. Blenski. Phys. Rev. A, 62:063204, 2000.

[4] C. Siedschlag and J. M. Rost. Phys. Rev. Lett., 89:173401, 2002.

[5] C. Jungreuthmayer, M. Geissler, J. Zanghellini, and T. Brabec. Phys. Rev. Lett., 92:133401, 2004.

[6] W. Ackermann et al. Nat. Phot., 1:336, 2007.

[7] P. Emma, R. Akre, J. Arthur, R. Bionta, C. Bostedt, J. Bozek, A. Brachmann, P. Bucksbaum, R. Coffee, F.-J. Decker, Y. Ding, D. Dowell, S. Edstrom, A. Fisher, J. Frisch, S. Gilevich, J. Hastings, G. Hays, P. Hering, Z. Huang, R. Iverson, H. Loos, M. Messersch midt, A. Miahnahri, S. Moeller, H.-D. Nuhn, G. Pile, D. Ratner, J. Rzepiela, D. Schultz, T. Smith, 
P. Stefan, H. Tompkins, J. Turner, J . Welch, W. White, J. Wu, G. Yocky, and J. Galayda. Nature Photon., 4:641, 2010.

[8] R. Neutze, R. Wouts, D. van der Spoel, E. Weckert, and J. Hajdu. Nature, 406:752, 2000.

[9] Z. Jurek, G. Faigel, and M. Tegze. Eur. Phys. J. D, 29:217, 2004.

[10] S. P. Hau-Riege, R. A. London, and A. Szoke. Phys. Rev. E, 69:051906, 2004.

[11] M. Bergh, N. Tîmneanu, and D. van der Spoel. Phys. Rev. E, 70:051904, 2004.

[12] B. Ziaja, A. R. B. de Castro, E. Weckert, and T. Möller. Eur. Phys. J. D, 40:465, 2006.

[13] C. Gnodtke, U. Saalmann, and J.-M. Rost. New J. Phys, 13:013028, 2011.

[14] B. Murphy et al. Submitted.

[15] Z. Jurek, B. Ziaja, and R. Santra. XMDYN, 2013. CFEL, DESY, Hamburg, Germany, Rev. 1.0360

[16] J. de Vries, H. Steger, B. Kamke, C. Menzel, B. Weisser, W. Kamke, and I.V. Hertel. Chem. Phys. Lett., 188:159, 1992.

[17] M. S. Dresselhaus, G. Dresselhaus, and P. C. Eklund. Science of Fullerenes and Carbon Nanotubes. Academic, San Diego, 1996.

[18] E.g. Petra III P04 XUV beamline, . http://photon-science.desy.de/facilities/petra_iii/beamlines/ p04_xuv_beamline/unified_data_sheet_p04/index_eng.html.

[19] M. Hoener, L. Fang, O. Kornilov, O. Gessner, S. T. Pratt, M. Guehr, E. P. Kanter, C. Blaga, C. Bostedt, J. D. Bozek, P. H. Bucksbaum, C. Buth, M. Chen, R. Coffee, J. Cryan, L. F. DiMauro, M. Glownia, E. Hosler, E. Kukk, S. R. Leone, B. McFarland, M. Messerschmidt, B. Murphy, V. Petrovic, D. Rolles, and N. Berrah. Phys. Rev. Lett., 104:253002, 2010.

[20] C. Buth, J.-C. Liu, M. H. Chen, J. P. Cryan, L. Fang, J. M. Glownia, M. Hoener, R. N. Coffee, and N. Berrah. J. Chem. Phys., 136(21), 2012.

[21] A. Reinkoster, S. Korica, B. Langer, S. Cvejanovic, J. Viefhaus, and U. Becker. K-shell plasmon excitation in $\mathrm{C} 60$ with subsequent double Auger relaxation, 2003. http://hasyweb.desy.de/science/annual_reports/2003_report/.

[22] D. W. Brenner. Phys. Rev. B, 42:9458-9471, 1990.

[23] Y. Yamaguchi and S. Maruyama. Chem. Phys. Lett., 286:336, 1998.

[24] S.-K. Son and R. Santra. XATOM - an integrated toolkit for x-ray and atomic physics, 2011. CFEL, DESY, Hamburg, Germany.

[25] Y.K. Kim and M.E. Rudd. Phys. Rev. A, 50:3954-3967, 1994.

[26] B. Ziaja, R. A. London, and J. Hajdu. J. Appl. Phys., 99, 2006.

[27] S. Korica et al. J. Phys: Conf. Series, 388:022087, 2012.

[28] J. Karvonen, E. Nommiste, H. Aksela, and S. Aksela. J. Chem. Phys., 106:3466-3472, 1997.

[29] F. Blanco and G. Garcia. Phys. Lett. A, 317:458-462, 2003.

[30] P. Juranic. Private communication. 